\title{
Self-control and Mental Health? Exploring Perceptions of Control from the Experiences of Black and Minority Communities
}

\author{
Dr Stephen Joseph* and Professor Frank Keating \\ The Royal Holloway University of London
}

*Corresponding author email: stephenjoseph19@gmail.com

Received: 19 December 2020 / Revised: 13 March 2021 / Accepted: 29 March 2021 / Published: 03 April 2021

\begin{abstract}
Although the disparities in mental health outcomes for ethnic minorities are well-documented, very little is known regarding service user perspectives and experiences from locus of control ambit. The aim of this qualitative study was to investigate the experiences of African and Caribbean service users, from the context of their Locus of Control (LOC). Informed by an Interpretivist approach, this study was conducted via in-depth interviews $(\mathrm{N}=10)$ and two focus groups $(\mathrm{N}=14)$. Purposive sampling methods were used to recruit mental health service users from a South London Borough to participate in the study. Data was collected through a semi-structured interview schedule and a focus group guide. Data analysis was informed by an Interpretive Phenomenology Analysis approach in the examination of the LOC profiles and service user perspectives. The study found that the drivers of LOC for the respondents were: perceived racism; perceived loss of control due to challenges pertaining to accessing employment, work stressors, lack of career advancement, socio-economic challenges; discrimination at work, identity challenges and entry to and exit pathways from mental health services, all of which promotes LOC externality. The findings suggest that mental health services need to take an active interest in factors that constitute externality in the LOC in assessment of the needs of A \& $\mathrm{C}$ groups in clinical and community interventions. A holistic approach to psycho-social and socio-cultural issues are highly needed to improve the mental health outcomes for members of African and Caribbean communities with experience of mental health challenges.
\end{abstract}

Keywords: Mental health; Ethnicity; Locus of Control.

\section{Introduction}

Ample research literature exists on mental health (Fernando, 1998; Keating et al, 2002), ethnicity (Fernando, 2009), the cultural paradigms of collectivism-individualism, (Cheng et al, 2013) and Locus of Control, (LOC), which is defined as the extent to which individuals perceive that they have control over events that impact their lives (Papadopoulos, 2009; Morling and Kitayama, 2008; Cheng et al, 2013). There is also extensive research into the inequalities in mental health for Black and Minority Ethnic (BME), particularly African and Caribbean (A \& C) communities in the United Kingdom (Care Quality Commission, 2014; Crisp et al, 2016; Keating, 2016); however, there are no clear-cut explanations (Department of Health, DoH, 2013), or strategic direction towards reducing such inequalities (Crisp et al, 2016). This study explored this issue from a different perspective by addressing the dearth of enquiry into possible connections between these concepts as a possibly useful explanation and action model towards addressing the inequalities relating to A \& C mental health. It was also intended to use the concept of LOC to explore possible answers to the current reported poor mental health experiences, profiles and negative outcomes of the subject population, and with a view to arriving at some suggestions to improve the current status.

Mental health service users are impacted by various factors such as social, financial and economic stressors which impacts on the development of their illness (Mantovani et al, 2017; Fearon et al, 2006). It has also been observed that "social disadvantages including unemployment; lone parent status; lower social class; low perceived social support; poverty..." and other issues contribute to the development of psychosis 
(Fearon et al, 2006: 1547). It has also been stressed that strong connections exist "between deprivation, ethnicity, and mental illness" (Edge, 2013: 40), as has also been found with the recent outbreak of Covid19 in 2020 and its disproportionately impact on BAME communities in comparison to their White counterparts (Smith et al, 2020; Iqbal, 2021; Proto \& Quintana-Domeque, 2020).). Consequently, health interventions need to focus on these elements, exploring their ramifications for each client to achieve positive outcomes (Elder et al, 2009; Engel, 2004; Borrell-Carrio, et al., 2004). By implication, research into this field also needs to apply the approach (Garcia-Toro and Aguirre, 2007).

An integral part of considering the psychosocial aspects of mental health service users includes the need to explore the link between their cultures and their perceived LOC in their life experiences (Cheng et al, 2013). An association between the social-cultural backgrounds and the perceived LOC of mental health service users has also been raised as highly important in their responses to services and overall journeys in mental health contexts (Cheng et al, 2013). Literature also posits that consideration needs to be given to the individualist-collectivist paradigm, as it highlights the differences between cultures (Papadopoulos, 2009). The concepts of LOC and individualism and collectivism (Morling and Kitayama, 2008) are explored and expanded upon in LOC across cultures and etbnicity - Individualism and Collectivism (Joseph, 2018).

The abovementioned has explored some areas that have been yet unexplored in relation to the challenges of BME mental health and the A \& C populations. It highlights that in spite of indicators from literature suggesting the need for it, there is a paucity of research which seeks to explore the concepts of collectivism as it relates specifically to $\mathrm{A} \& \mathrm{C}$ people in mental health contexts.

The mental health experiences of the BME and A \& C groups, in particular, have also not been explored using the lens of LOC from the domain of the likelihood that their LOC can be influenced by their culture and ethnicity in mental health contexts. For example, it has been cited that one of the potential explanations for a low level of help-seeking for perinatal depression by women of Black Caribbean ethnicity was due to their "beliefs about mental illness, their fear of stigma, and their help-seeking approaches - central to which is a strong external LOC and self-reliance" (Edge, 2011: 256). This findings by Edge (2011) corroborates the findings from earlier studies into mental health LOC and Locus of origin, which delves into beliefs regarding the aetiology of mental illness, that these beliefs play a role in the LOC, attitude and responses of individuals, including patients and health professionals in mental health contexts (Hill and Bale, 1980; Cheng et al, 2013).

This further highlights the need to investigate this gap in knowledge by conducting an in-depth exploration of LOC from the A \& C mental health service users' perspectives in general and mental health contexts. This is in order to discover their LOC profile as groups with experience of mental illness, as well as factors that may be impacting on their LOC in relation to their mental health experiences. Recent findings have also highlighted the significance of and the dire need for incorporating perceived control from a domain of culture in the conceptualisation of models, designing research, and intervention programs for mental health (Cheng et al, 2013).

This study explored the perceptions of members of A \& $C$ populations with experience of mental health services on LOC in general and mental health in the context. The qualitative study was conducted in 2 stages: a) in-depth interviews and b) focus group interviews, with mental health service users in a South London Borough. This was with a view to using the lens of LOC to assess what the respondents perceive to play significant roles in their mental health experiences and services. The idiographic and hermeneutic approach applied by Interpretive Phenomenological Analysis (IPA) in exploring detailed account of respondents' experiences and 'world view' makes it highly suitable for this study (Smith et al, 2012).

\section{Methods}

\subsection{Participants}

As shown in the Table 1 (Demography of study sample), a total of ten mental health service users who selfdescribed as being of African and/or Caribbean origin participated in one-to-one interviews while a total 
of fourteen men and women meeting the criteria participated in two focus groups in this study. This followed approaching various service users who were participating in various drop-in sessions in the charity organisations, with the support of the coordinators who were familiar with the participants. In line with purposive sampling, those who volunteered to participate out of approximately sixty individuals approached by the researcher were given information regarding the study, and the criteria for inclusion. Many of the service users who indicated their interest and met the inclusion criteria were however unable to participate due to availability and time constraints. Others who indicated that they were not comfortable to participate in one-to-one interviews volunteered to participate in focus group sessions, for personal reasons.

The ten participants in the one-to-one interviews consisted of six women and four men whose ages ranged between 36 and 58 years (mean age of 47). Eight respondents participated in the first focus group session comprising of five women and three men while the second focus group session was attended by six participants made up of three men and three women. The age of participants in both groups ranged between 32 and 55 (mean age 43.5). None of the participants were in paid employment nor were any participating in any volunteer work or study. All the participants had experiences with mental health services in a variety of forms and over a long period, including inpatient admissions and community mental health services including case management. The diagnoses reported by participants included Schizophrenia, depression, bipolar disorder, anxiety disorders, and schizoaffective disorder. All the participants were receiving a government disability pension at the time of the data collection. None of the participants were married while only one reported to be living with a partner at the time of interview. One was a widow with grown children while no other participant reported having any children. Four participants (two male and two female) reported to be volunteering with charity organisations, three of which involved acting as peer supporters with people living with mental illness, while one of them was supporting someone with a learning disability.

Table 1: Demography of study sample

\begin{tabular}{|c|c|c|c|c|c|}
\hline \multicolumn{3}{|c|}{ One-to-one interviews x 10} & \multicolumn{3}{|l|}{ Focus groups $x 2$} \\
\hline Variable & Results & Percentage & Variable & Results & Percentage \\
\hline $\begin{array}{l}\text { Gender } \\
\text { Male } \\
\text { Female } \\
\text { Age }\end{array}$ & $\begin{array}{l}4 \\
6 \\
\text { Range } \\
36-58\end{array}$ & $\begin{array}{l}40 \% \\
60 \% \\
\text { Mean } \\
47\end{array}$ & $\begin{array}{l}\text { Gender } \\
\text { Male } \\
\text { Female } \\
\text { Age }\end{array}$ & $\begin{array}{l}6 \\
8 \\
\text { Range } \\
32-55\end{array}$ & $\begin{array}{l}42 \% \\
58 \% \\
\text { Mean } \\
43.5\end{array}$ \\
\hline $\begin{array}{l}\text { Marital status } \\
\text { Single } \\
\text { Married/cohabiting }\end{array}$ & $\begin{array}{l}9 \\
1\end{array}$ & $\begin{array}{l}90 \% \\
10 \%\end{array}$ & $\begin{array}{l}\text { Marital status } \\
\text { Single } \\
\text { Married/cohabiting }\end{array}$ & $\begin{array}{l}13 \\
1\end{array}$ & $\begin{array}{l}93 \% \\
7 \%\end{array}$ \\
\hline $\begin{array}{l}\text { Ethnicity (self- } \\
\text { described) } \\
\text { Caribbean } \\
\text { African }\end{array}$ & $\begin{array}{l}6 \\
4\end{array}$ & $\begin{array}{l}(60 \%) \\
(40 \%)\end{array}$ & $\begin{array}{l}\text { Ethnicity (self- } \\
\text { described) } \\
\text { Caribbean } \\
\text { African }\end{array}$ & $\begin{array}{l}9 \\
5\end{array}$ & $\begin{array}{l}64 \% \\
36 \%\end{array}$ \\
\hline $\begin{array}{l}\text { Employment status } \\
\text { Employed } \\
\text { Unemployed }\end{array}$ & $\begin{array}{l}0 \\
10\end{array}$ & $\begin{array}{l}- \\
100 \%\end{array}$ & $\begin{array}{l}\text { Employment status } \\
\text { Employed } \\
\text { Unemployed }\end{array}$ & $\begin{array}{l}0 \\
14\end{array}$ & $\begin{array}{l}- \\
100 \%\end{array}$ \\
\hline
\end{tabular}

\subsection{Data collection}

Ethical approval was obtained from the Royal Holloway University of London Ethics committee (Ref. number 15072015). Informed consent was given by all participants after Participant Information Sheets (PIS) were given and explained to them, assuring them of ethical principles including protection of their 
right (Prince et al, 2003), avoidance of harm or distress in the course of collection, analysis, and dissemination of findings, guarding their autonomy, confidentiality and anonymity (Redsell and Cheater, 2001), and fidelity (Ruiz-Casares, 2014). The one-to-one and focus group semi-structured sessions took place in private at the premises of charity organisations at the convenience of the participants. The one-toone interviews lasted between forty-five minutes and one hour while the two focus groups sessions lasted between fifty and seventy minutes. All the sessions were digitally recorded in audio format and transcribed verbatim. Participants were also given pseudonyms in the report. Although this study involved participants with previous experiences of inpatient mental health admissions, the ethics committee found that a National Health Services (NHS) ethics approval was not required as it did not involve collecting data from participants in NHS inpatient settings or in NHS patient facilities. However, the Caldicott principles for handling personal data as it related to health service users (National Health Services, NHS, 2013) were observed. This includes that data be processed: fairly and lawfully; for specified purposes; adequately, relevant and not excessive; accurate and up-to-date; not kept for longer than necessary; observing the rights of data subjects; protect by appropriate security; and not transferred outside the European Economic Area (EEA) without adequate protection (NHS, 2013). Scientific and ethical rigour was also upheld in accordance with the Helsinki declaration (Prince et al, 2003) throughout the study.

Although the findings from the ethics committee indicated that the research did not pose any serious ethical issues, the researcher was mindful of the likelihood that sensitive issues might arise during the interviews, as the participants were recalling their past mental health experiences. Efforts were made by the researcher with the support of the coordinators of the charity organisations to conduct gatekeeping by debriefing the participants after the interview sessions. This was in order to ensure that any emotional challenges or recall of trauma they might have experienced during the interviews were discussed, and to offer them support if required.

Two interview schedules were developed for this study; one for the individual interviews, and the other a guide for the focus groups (Appendices 4 and 4 respectively). The interview schedule asked participants what constituted control in their lives from their mental illness experiences contexts. Participants were also encouraged to express their sense of control from both before and after their experiences of mental health challenges, as well as their experiences with services. The interview questions were applied with flexibility in order to further explore their responses in a non-predictive way. The questions were initially piloted with a group of four African and Caribbean mental health service users in order to obtain feedback on the content and structure of the questions and gain insight into the adequacy and feasibility of the instruments (Teijlingen and Hundley, 2002; Arain et al, 2010; Thabane et al, 2010). The opinions expressed by the four A \& C service users were incorporated into the final version of the two sets of questions designed and used for the study.

Following a successful pilot, the interviews were conducted with attention to the interviewees' responses to the semi structured questions. Follow-up questions were also asked to further explore the interviewee's responses to particular questions, in order to elicit detailed information that promotes gathering of contextbased data relating to the subject matter of the participants' perception of control (Williams and Katz, 2001; Smith and Osborne, 2003). Concerted efforts were made by the researcher to limit interview bias and the likelihood of individual voices being silenced in a group situation in the focus group sessions (Kitzinger, 1995), ensuring that every focus group member was heard, and that group members' conversations were focused on the main purpose of the study (Willig, 2003; Starks and Trinidad, 2007). The moderation also involved asking additional questions for further clarification, observing the atmosphere and interaction among participants and making brief notes regarding the two sessions. Attention was also paid to acknowledging the individuality of each focus group as a unique set of opinions (Stewart and Shamdasani, 1990), and aim to promote generalisation with the complimentary tool of individual interviews. The twopronged approach of combining focus groups and one-to one interview was also adopted in order to take advantage of the benefits as well as minimise the impact of the demerits of both approaches (Lambert and Loiselle, 2008), including complimentary and overlapping findings that can contribute to "a coherent and 
more nuanced understanding" of phenomenon being studied (Lambert and Loiselle, 2008: 234). All interviews and focus group sessions were audio recorded digitally with the consent of all participants and later transcribed verbatim for analysis (Brocki and Wearden 2006).

\subsection{Analysis}

Analysis of the data was conducted following an idiographic approach, in line with the Interpretative Phenomenological Analysis (IPA) procedure (Smith et al, 2012). This involves an iterative process of reading and analysing the interview transcripts in sequence by moving in the order of repeating the cycle of describing as well as interpretation (Smith et al, 2009; Brocki and Wearden, 2006). After going through each transcript on two occasions, notes of key words identified as used by the interviewees to express their experiences and opinions were initially made on the left-hand margin of the transcripts. This is to ensure that the language used by the participants were noted and considered in the interpretation. This was followed by stating the researcher's interpretation of the key words on the right-hand margin (Clarke, 2009). The two focus group transcripts were also analysed using the same iterative process involving sequentially analysing, describing, and interpreting, with notes of identified key words and commonality of ideas between the participants (Clarke, 2009).

It was found that different patterns of information emerged from the two focus group settings, as the participants' responses were presented in both individual and general contexts, with examples from their individual experiences as well as their observations in the course of their experiences. The interpretations of the views, experiences and examples were then converted into a list of ideas that could be considered as themes with the use of memos and tables set up by the researcher to ensure that all the ideas were accounted for. These themes were explored further as overarching, meta, master, (superordinate) and subordinate themes (Griffiths et al, 2011; Mulveen and Hepworth006; Knight et al, 2003; Smyth et al, 2011).

All the themes found for each transcript were gathered along with the respective quotes that support the themes, following which the transcripts were further explored to gain familiarity as well as achieve some level of assurance that some issues raised by the participants have not been overlooked. This enabled the researcher to conduct a comparison of themes identified from all the ten interviewees and the focus group participants; and to be able to identify the themes that have a strong commonality and weight amongst all the participants. The triangulation of the focus groups and one-to one themes was also conducted to identify areas in which the combination of both methods has led to increased understanding from the responses to the interview schedules and focus group guide from individual and group sources. It was also observed that many of the observations, experiences and views expressed in the focus group settings were similar to some of the views and experiences found in the one-to-one transcripts. In addition, it was found that additional information emanated from interactions and discussions of experiences in the focus groups that were not indicated in the one-to-one interviews, which suggests that the focus group settings also contributed to a higher level of understanding of the responses received. The second stage in the analysis involved presenting the identified themes to a supervisory researcher for further in-depth look and advice, in order to ensure that methodological rigour and quality assurance were observed at this stage (Darawsheh, 2014), as it was also found that the combination of the total of twelve transcripts resulted in more issues raised in comparison to relying on only the one-to-one interview transcripts. The advice and directives resulted in the principal themes and subordinate themes for the whole dataset which were considered a fair representation of all the themes identified, (some of which were combined for ease of data management), that highlighted issues to be further explored in the interpretation (Smyth et al, 2011).

\section{Results}

The accounts of participants regarding what control meant for them and their experiences with mental health challenges were analysed using IPA. Two key superordinate themes were drawn from the analysis, they include: Meaning of control and Levels of Control in relation to mental health experience. A summary of the findings can be found in Table 2 . 
Table 2: Superordinate and subordinate themes emerging from the results

\begin{tabular}{|l|l|}
\hline $\begin{array}{l}\text { 1.Perception of Control- } \\
\text { mental illness triggers }\end{array}$ & $\begin{array}{l}1.1 \text { - Stressful factors } \\
1.2 \text { - Identity challenges }\end{array}$ \\
\hline $\begin{array}{l}\text { 2.Perception of Different } \\
\text { Levels of Control in relation } \\
\text { to mental health } \\
\text { experiences }\end{array}$ & 2.1 Admission process and hospital experiences \\
\hline
\end{tabular}

\subsection{Perception of control}

Participants expressed what control means for them from the ambits of factors they identified to have triggered mental illness in their experiences- stressful factors and identity challenges.

\subsubsection{Stress factors as mental illness triggers}

Under this sub theme, most participants expressed their view on the meaning of having control over one's life by narrating their experiences prior to what they described as a 'mental breakdown'. They likened being in control over their lives to the conditions of their lives prior to the commencement of the experiences that they expressed caused stressors to their daily living. They attributed a loss of control of their lives to the stressors that they identified as challenges to their mental health which led to hospitalisation. Some participants also attributed this perceived lack of control of their lives due to the stressors and conditions as factors which led to their mental illness experiences.

One aspect of this lack of control was expressed in relation to the experience of stress at work and challenges with coping with work demands. Alison described a strain of work demands including long hours of work:

"From work... It was a strain. I used to do a lot of hours, used to start at 7 in the morning and finish at 9 at night and it's catering and it's constant..... all the time". (Alison, 1-1)

Carla also described the experience of breakdown due to long hours of work, also expressing a surprise that she survived:

“.....it lead to the breakdown... umm.... w was doing a lot of hours and I wasn't eating well and um... it was a constant thing of worrying all day and you don't realise the pressure. I'm still shocked that I'm still here". (Carla, FG2)

These suggests that the stressors of work demands and their impact on the feeling of control or a feeling of not being able to cope with the demands of work may constitute an externality in LOC, as the participants could have experienced lacking control over their lives as a result of those stressors. As these pressures and demands of the daily routines contributed to mental health challenges for most of the participants, it would also suggest that a feeling of not being able to cope could have been considered a sign of failure leading further to externality in their LOC.

Another aspect of the stressors that were expressed by most of the participants as having led to them feeling a lack of control over their lives, and which led to mental health challenges was the pressure felt from their superiors in addition to work demands. For example, Patricia described having one's 'structure' being used all day, and the action of a 'boss':

"It was a pressure because sometimes you had to be on your own and work on your own and there was a lot of uhm.... deliveries and stuff like that to do and you know your whole structure is being used all day......... well.... someone tends to be more of a boss than you are. You know, stepping all over you, inconsiderate". (Patricia, FG1) Some of the participants also perceived that a feeling of having their lives being taken over by someone else, rather than their own actions (external LOC), as having contributed to their mental illness experience. 
An example of this perception is contained in Alison's description of her experience at work in which she expressed feeling that someone else had taken over her life:

'I couldn't say the lead to this was my own actions, I couldn't say that. Like if it's my own actions it means that someone took over and I wasn't around. Simple" (1-1).

Having a feeling of someone else taking over one's life is also an expression of externality in LOC, as indicated in Alison's statement which she perceived that she 'wasn't around'.

Some participants also expressed losing control over their lives due to the perception that their lives were being controlled by others. Brian explained that he felt that addictions, including alcohol and drugs played a part in his view of not being in control:

'In the past it's been all external, a lot of external things have controlled me....erm...well I didn't have control over my life because of my addictions. My life was unmanageable basically and my mental health...it was unmanageable... (due to) ... alcohol and drugs" (FG1)

The expression of the perception of loss of control was also explained by many of the participants as being due to a combination of factors including pressure in the workplace due to experiencing exhaustion, frustration, discrimination and a perceived lack of promotion, and perceived poor prospects in career advancement. One example of these factors is contained in the statement by Patricia who described a feeling of 'being suppressed', a feeling of low prospect for career progression in spite of being told that she possessed the necessary skills and ability, she was not considered for managerial roles, which she further described led to 'burnout':

".....the fact that I was being supressed in the work place and erm.... w was always told that I had the skills and ability but promotion was erm.... what they call that glass ceiling and it just wasn't happening. I would be erm...you know... in the presence of absence or in the....you know... anything like that; I would be called upon to carry out the role but actually having the role in a full time capacity wasn't going to happen. It became disheartening and it leads to.... it led to burnout really... that's the...that's the correct diagnosis for it. It was burnout".

This experience was also expressed in relation to the consequences of those factors which were considered frustrating by Patricia, which then led to mood instability, cognitive difficulties, and mental illness:

'Burnout leads to severe depression....severe depression leads to unable to control one's thoughts, one's mood, one's overall judgement; everything just kind of......... Because it's a ...it's a grinding down. It doesn't happen overnight it's a continuous grinding down of the person's strength until the person is unable to function and then....you end up severely ill" (FG1).

These suggest that the feelings of these factors were indicative of a lack of control and externality in LOC, which led to mental illness for many of the participants who were working before they became mentally unwell.

The expression of the attribution of racial discrimination as precursor to and contributory to their experience of mental illness was also a constant feature in most of the participants' description of what it meant to lack control over one's life. An example of this position is indicated in Mary's statement in which she described experiences of 'racism and prejudice', of being 'over the edge' which resulted in 'breakdown', and having to stay with a friend and engage with a psychiatrist:

"But the major thing that really, really sent me over the edge... remember racism and prejudice based on culture and belief....... just doesn't start at one point. It starts from the year dot, it's continuous; and it breaks you down and it breaks you up inside and it mashes up your head.... he phoned my friend and she came and got me in the pub, and I stayed in bed for about 2 weeks in her house and that was the real start of what I call my breakdown. That was it from there and from there I had to go and see a psychiatrist, the doctor came and take me to the psychiatrist...". (1-1)

The experience of racism was also expressed as being contributory to a lack of career advancement. For example, Carla expressed being denied opportunity because of her skin colour, and that advancement in her career would have been achieved if she had not been discriminated against in the workplace:

"Well I actually feel that if I had have been a white British...I would have made it because ...I know and I know that because I was there. So I erm.I feel that based.... I was denied an opportunity based on my skin colour". (FG2) 
The experience of racism was also expressed in the parlance of leading to frustration and unemployment as a result of not being able to cope and having to take the decision to leave employment. An example of this lack of control due to perceived discrimination on account of race was expressed by Alan who described leaving his employment due to racism:

"Well my reaction was gradually erm... frustration and annoyance and stress and erm...gradually.....eaving the post". (1-1)

The loss of employment due to racism could have in turn led to further loss of control of one's life's finances and contributed to their breakdown. These are also indicative of externality in LOC.

The experience of racism as being a contributory factor in expression of a lack of control was also discussed by many participants from the context of their childhood experiences. Many expressed that experiencing racial abuse during childhood led to a feeling of a lack of confidence and self-esteem, and control over their lives. Furthermore, the majority of these participants attributed their mental health challenges to these past racial abuse experiences. An example of this view is contained in Alison's statement in which she described experiencing racism, and suffering from anxiety when young:

"... I had a lot of racist, I had a lot of racial problems and erm..... had a lot of anxiety. I suffered from anxiety from a young age". (1-1)

Brian also expressed having to report to his family his experience of racial abuse when he was growing up:

"Well how I was brought up, the way I was brought up; because I had so much abuse... racial abuse.I would come in crying to my family saying "someone called me a blackie or a nigger" or something like that. I used to have terrible things said to me" (Brian, FG1).

The experience of racism and bullying during younger years was also expressed as having impacted on the feeling of a loss of control over one's life by many of the participants. This was also expressed as a factor that led to mental health challenges in later years, even as the majority of the participants also described having experienced some form of mental health challenges from a young age. An example of this view was Carla and her experience of bullying and racism which led to anxiety, resulting in being placed on medication at a 'young age'. She also expressed a feeling of being treated as just a 'young black girl', a lack of empathy and not being offered counselling:

"From that young age I was on medications and the way I was treated was like "oh she's just a young black girl" and this and that "she'll be alright". There was no counselling, there was no empathy, there was no understanding of who I was or what was going on for me; anything like that and the reason why I was anxious. The reason why I was anxious was because I was being bullied and being taunted and you know racial things and stuff like that. Their answer was to give me medication and that would take away the anxiety". (FG2)

A similar experience was expressed by another participant, as being contributory to their mental illness experience. This was also illustrated in the context of how the causative experience has extended to their feeling a loss of control and a sense of dependency after discharge from hospital:

“"Well essentially my mental illness has been caused by predominantly traumatic events in my life. Bullying at school, mistreatment by mother's neighbours and stuff like that ..." (Dwayne, 1-1)

These expressions and the accompanied sample statements indicate that the participants were predominantly undergoing a high level of loss of control over their lives as a result of the various stressors outlined. It suggests also that their LOC were on the high externality scale, which led to their mental illness experiences as well as contact with the services. The expressions of experiencing 'frustration and annoyance and stress', 'bullying at school', 'being taunted' and references to 'traumatic events' are likely factors that could lead to a perception of a loss of control in one's life. It thus suggests that the majority of the participants were placed in the externality end of the internal-external LOC spectrum before their contact with the services. It also indicates that some of them were also fallen within the externality spectrum from a young age as a result of the experience of racism. 


\subsubsection{Loss of Identity}

Most of the participants also expressed the role played by a perceived loss of identity in their perceived loss of control over their lives. They adduced a sense of identity loss as a factor that did not only act as an integral part of their mental health challenges, but also carried through into their mental illness experiences. Participants explained that having been born and growing up in Great Britain, and failing to find creative avenues and "services" that would enable them to explore their interests as men and women of A \& C backgrounds, also led to confusion regarding where they belonged, frustration, anger and a feeling of a lack of cultural identity. An example of this perception is included in Mary's statement, which included a feeling that she was also controlled:

'Well I was a very confused person, I had no identity; I didn't know my identity and I was controlled. My people and the authorities controlled me, I had no say, I had no say". (1-1).

This also suggests experience of a challenge with having a sense of belonging, as it has also been posited that identity, which describes how one knows oneself and how one is known by others as well as identifying with groups, are important to mental health (Haslam et al, 2009). This challenge was also described by Brian who expressed making effort to find his own identity, but which was hampered by his struggle with his mental health challenges:

"I felt it was very important for me to formulate my identity but at the time I was half in balf out in terms of my own mind because I was struggling with mental illness at the same time and I didn't know it you know". (1-1).

In another example, Ryan (1-1) described a perceived loss of identity through feeling isolated, due to a lack of 'services' he could access as to explore his creativity, playing a role in his mental illness experience:

“.... contributed to me having a mental health issue...my experience was umm....ummm... I lost control. I lost control of my identity. I had my identity in the days when I was growing up but somewhere along the way when I grew up. I started to mix a bit more at my own pace in the community. I did get isolated at one time because services at that time wasn't around. The services that I wanted to obtain weren't around. I had to make do with whatever was available which wasn't what I was looking for so....erm...I was looking for something a little more creative, something a little more outstanding, something that could help people.." (1-1)

Alan also described his struggles with finding facilities that could enable him to explore his interests and find his identity, and the view that he did not receive the support he needed. In citing the example of the level of education he had attained to affirm to himself that he was not 'stupid'; he also expressed that he felt that he was treated 'like a child' instead of being provided the support he was looking for:

'It's very difficult you know, you know I have got two degrees in my time. One in law and one in biomedical science, which I didn't complete for various reasons, because I became ill or gave up for various reasons. So what I'm trying to say is I'm not stupid. So it's really difficult to find facilities who really cater for me you know, in terms of my identity, my interests, you know they were trying to be nice to me but I remember they were talking to me like a child you know? I was 38 years of age. People are affected differently and people react differently you know" (1-1).

The loss of identity was also linked with the role culture played in the perceived loss of control that was expressed by most of the participants. In expressing their hospital experiences, the majority of the participants also linked their view of a lack of control from the context that their culture which was part of their identity was not acknowledged. For example, Adrian described the impact of his hospital experience in relation to having nothing to remind him of his culture. He also explained the importance of culture as the 'root' of himself, how he becomes different without it, describing that as 'someone else', 'a different me', as well as its importance to his identity. He also expressed a desire to gain his identity again, and recognise his heritage and tradition:

"Whilst I was locked up in the hospital on the ward there was nothing there and no one there to remind me of my culture. As I said, the root of myself is my culture and if the root is not there, it's not me, it's someone else; it's a different me. So the root has to come back for me to grow again and to gain my identity again and to recognise my beritage and tradition". (FG1) 
The foregoing indicates that most of the participants perceived that their LOC were highly external due to the influence of their perceived loss of control. This perception was also expressed as being due to their view of a loss of identity, the lack of control over their identity, as well as a lack of support to enable them explore their identity. This includes a perceived lack of creative avenues as well as services through which they could be able to explore and regain the identity they perceived to have been lost. This perception also seemed to be exacerbated by their mental health challenges. They also expressed their hospital experiences in which they reported that they were unable to find things and people to which they could relate with culture; this appears to have also contributed to their feeling of a loss of their identity as their culture was integral to their sense of identity. These expressions confirm the literature position that a feeling of that one is not able to exert an influence on issues in one's life is an indication for externality in LOC.

\subsection{Differences in perceptions of control - pre and post hospital experiences}

Within this subtheme many participants related their perception of control to their experiences with services during their mental health journeys. It contains their views regarding control over their lives from two different contexts- hospital admission and stay and their post discharge experience. It comprises of the expression of the sense of control they perceived during their hospital admissions, including the process that led to their hospitalisation for treatment, their hospital stay on the one hand, and their perception of LOC following their discharge from hospital on the other. The various stages of experiences were expressed by most participants as being different from each other, which in turn placed their perceptions on different levels of LOC.

\subsubsection{Admission process and Hospital experience}

Most of the participants' description of their hospital experiences were characterised by a feeling of a lack of control. This includes the admission process in which many of them expressed that their opinions were not taken into account. An example of this view is included in Dwayne's narration of his first experience of admission which involved the police. He described having no control but experience of having his door 'kicked down', being accused of doing things and then being given injection against his will:

'The first time they put me in hospital they took me in there in my underwear... they wouldn't even me let me put my clothes on when they came. They just kicked the door down and just rushed in and they just started talking all this rubbish about all these things I didn't do...... Then I was in the shower with the door kicked off and I said to them "well if you're going to take me away I expect you to let me put my clothes on and you can take me away. They took me out of the place in my underwear, put me in the unit, took off my underwear, gave me an injection, I fell asleep and woke up on the ward...." (1-1).

The view of a lack of control (external LOC) indicated in not being able to have their view expressed or taken into consideration also appear to be further exacerbated by the hospital stay experiences, as the participants' sense of lack of control were described from the ambit of having no say or any control over decisions. Alan's statement includes an example of this view in which he narrated being taken away when his mother called a doctor to 'examine' him, which was then followed by police being called, and things happening 'so fast'. He also indicated that he was 'still trying to have a say':

"On my admission to the mental health hospital, which was some years ago now I had no control over it, I had no say in it at all. A short excerpt from my story is that I was at my mum's place having dinner and she called the doctor in to examine me, the police came and they took me in the ambulance to the mental health hospital. I didn't have a say in it all, everything happened so fast and the mechanism that was in place was that I didn't have a say in it and to this day I'm still trying to have a say in it even though it all happened a long time ago. So what I'm doing now to help with this PbD and this interview um... is of benefit to me as well umm.. to speak. up about what happened to me you know erm... and for someone to say well this is anful or this is good or this is something you know? Erm.... had no control". (1-1). 
This narrated experience which included an expression of a desire for someone to comment on their experience seem to be a need for a validation, and also seem to indicate that the participants still feel that their voices are not being heard even after a lapse in time since their admission into hospital.

These views expressed by the participants regarding not being in control during the admission process appears to have been exacerbated during their period of stay in hospital. Most participants expressed a perceived continued loss of control whilst in hospital, including a lack of involvement in decisions about their treatment. An example of this view in contained in Dwayne's statement in which he describes his experience of decisions being made on his behalf. He also indicated externality in LOC in his response to that experience, his way of stating his opinion without challenging decisions made for him in order to manage his situation, as felt that his perceived condition of a lack of influence on the decisions would not have changed if they had been challenged by him:

"... decisions were made for me and the only way I could influence them is by....is by....well, basically agreeing to what had been said and then coming back and saying my opinion on what that decision was. So, I did learn to.... I didn't really challenge any decisions because that wouldn't have helped the situation I don't feel. I was better off trying it, coming back and saying why I agreed with it or disagreed with it". (1-1).

Another participant also reported that the lack of say into the decision made by the health care professionals for them to commence a medication regime made them feel that they had lost control of their lives. An example of this view was expressed by Dennis' statement in which he described having 'no say', being forced to take medication, and feeling like a baby:

"I had no say in the medication they forced me to take. I was like a baby because of the medication which I called a spiritual strait jacket." (FG1)

This expression of externality in LOC in feeling of having a 'spiritual strait jacket' indicates that participants felt restrained in a spiritual sense, which could also be interpreted as feeling a loss of control both physically and spiritually. Noella used a similar expression of having 'no control' of her life in hospital. She also indicated that she found herself having no choice but to comply when 'decisions were made' for her. She also expressed that she felt that 'power came in' for her when she decided that she had to leave the hospital:

".. I had no control of my life when I was in hospital and decisions were made for me and I had to do that but power came in for me when I made the decision that I'm not staying in hospital. "I don't know what's going to happen, what's going to take place, I don't want to stay in hospital, I want to go home" I tried as best and I could and see the outcome..." (1-1).

The expression regarding power also suggests that the participant might have felt 'powerless' from the experience of having decisions made without her control or input, to the extent that she felt that she had to draw on 'power' (internality in LOC), by summoning courage to take the decision to leave the hospital, although she did not 'know what was going to happen'.

Another example of the view of externality in LOC due to a perceived lack of control is contained in Alison's statement in which she also described the impact of the experience of hospitalisation has had on her. She expressed finding it difficult to speak about it even after discharge, being stirred up, being unable to 'get over it', and having to 'walk around the streets just to make sure':

'It sits on you and stirs you up um... it's just hard talking about it because you're not that far from it...it's not like it happened, it took place and you get over it. You can't really get over it because there's so many things going on, so many things attached to it. From home to hospital it's different, just to...just to....I have to walk around the streets just to make sure, just to know... I'm around my place....I'm by myself that I'm not with people like I used to and I find I get tired but you know it needs to be... it needs to be with people that can explain" (1-1).

These expressions also seem to indicate that the level of externality in LOC had been so high to the extent that the participant felt that they had to be reassured that they were 'free' after being discharged. It also gives further indication that the majority of the participants described a difference in their perception of a lack of control between their experiences whilst on hospital admission in comparison to their experience when they were discharged. 


\subsubsection{Post hospital experience - contradictory levels of control}

In this subtheme, many participants described a feeling of gaining control over their lives after discharge from hospital. This sense of control was in the parlance of being able to make decisions. One example of this is contained in Jane's statement in which she also expressed that she could no longer be controlled, dictated to, as well as finding herself and her own identity:

'Well obviously I feel that I've found myself now and my identity and I feel that I'm able to speak up a bit more for myself now and things like that and they can't dictate to me.... they can't control me. They used to be able to do that in the past" (Jane, FG1).

These expressions suggest that the perceived loss of control within the hospital experience is also being linked to a perceived loss of self, and loss of identity as an African or Caribbean individual. This link also correlates with the expression of loss of identity or challenges regarding identity as being a trigger to the illness experience described under the perception of control themes.

Another expression of sense of control after discharge from hospital is also found in Carla's statement in which she described being 'pretty in control':

"Oh yeah, I feel as I'm pretty in control yeah, yeah....Erm.... with my mental bealth, with my physical bealth to a degree. I mean there are certain things that will come up with my physical health that it's just natural and wear and tear as they say. My housing situation is under control, my financial situation is under control. My day to day living is under control" (Carla, FG2).

This also indicates that a feeling of control of one's life is linked to having situations such as housing and finance 'under control', thereby further suggesting that a perceived control over these issues are indicators of LOC internality from these participants' perspectives.

However, majority of the participants also expressed that they later found that the perceived sense of control was short-lived, as they found themselves to be highly dependent on others for daily living activities and for their livelihood:

'To a degree I'm in control and in part I'm not in control because like I said it's this medication. If I come off this medication I'm scared. I don't know what would happen to me so I feel that I am controlled by the system to stay on the medication and to another extent I could try to come off the medication slowly with the help of the professional people..... and see what happens". (Carla, FG2)

Another participant also mentioned feeling a loss of control due to dependency on medication:

"To a degree that I am and to a degree I feel that I'm not because I'm on medication now permanently and I feel like I'm not in control and not in charge because I feel that the medication is in charge of me". (Tim, FG2).

One of the participants described a lack of control post discharge from hospital from a feeling of a sense of boredom and an inability to make sense of the world, and having to depend on other people:

“...but I ....with the boredom that's actually built up over my life over the years um....you still need someone talking to you, you still need someone that's normal ummm..making sense for you. You still need all that, you don't know what's sensible really, you don't know what's sensible". (Dwayne, 1-1).

In further response to the question regarding what it means to be in control of one's life, majority of the participants indicated that it was necessary to be able to cope with daily activities both physically and emotionally, be confident and financially independent. An example of this view of control over life is included in Tim's statement in which he described several factors to be accomplished, including financial, maintenance of physical and mental health, assertiveness and confidence in their relationship with health professionals:

'To be in control of one's life, we can live from day to day ... maintain our house if we've got one, maintain our life, maintain bills erm....maintain mental and physical health. Be able to socialise, be able to interact, be able to stand up for oneself when we need to, be assertive, be confident and let the doctors and the mental health team.... to be able to make them aware of who we are and our capabilities and needs and things like that and that we are in control',.(FG2). 
This seems to indicate that participants considered that some specific factors are prerequisite to being in control'. It also suggests that internality in LOC is linked with the ability of people to be able to achieve self-management including maintain a combination of financial, physical, mental, self-confidence, and social interactions. These, however, seem to be lacking in the description of most participants' experiences which included a feeling of powerlessness after discharge. An example of this experience is included in Dylan's statement in which he expressed that the long period of admission had led to him becoming confused and unable to decide on how to control his life's situation when he was discharged. He described a lack of people to socialise with, 'having no control', and a feeling of helplessness that what he expected to get support from the doctors did not 'seem to work':

"After such a long time in that place, having no control I felt....um....I was mad. I wasn't able to think about getting back. I've been brought this way and what am I going to do, what am I going to do? I don't know anyone, I just don't know. I expected to be in more with doctors and stuff like that but um... it doesn't seem to work that way" (1-1).

In addition, many of the participants also expressed feeling of a loss of control in relation to finances, and being dependent on the government for survival. For example, Melanie expressed an inability to gain control of her finances as well as regain her career due to the long time spent in hospital; she also described the impact of the medication resulting in making her feel like a 'zombie':

".... I mean, I was not in control, couldn't get any more career, and the psychiatrist, as I have said, those bloody tablets she gave me half killed me anyway. And, I ended up like a bloody zombie on benefits". (FG2).

These expressions indicate a high level of externality in LOC, including a feeling of helplessness, a loss of confidence due to long period of hospitalisation, a feeling of a poor prospect in regaining one's career, and a feeling of dependency.

The link between the post discharge experiences and the perceived lack of control was also expressed by the majority of the participants from the perspective of the need for the stressors that contributed to the mental illness experiences to be addressed to enable them to regain control over their lives. Furthermore, most of the participants expressed an inability to secure and retain gainful employment due to their mental illness experience and the associated ongoing stressors which impacted on their ability to secure gainful employment and maintain their mental health. For example, Alison's statement below described a scenario in which someone has received good mental health support but the benefit of which is hampered by what she described as 'those stressors still in the community':

"....so again going to a psychiatric hospital yes you're mended you're well, you're given all the support and the psychological help and you're on the right balance of medication but if those stressors are still in the community, unless you have developed coping mechanisms or a way to accept that or deal with it you're always going to be fighting against it and end up ill or you are just going to become subdued and accept what's happened so it doesn't seem like a win, win situation". (1-1).

This suggests that the impact of the challenges experienced in relation to social issues of unemployment and other social inequalities (Williams and Keating, 2005), are likely to impact on people's LOC in both general and mental health contexts, even if they had received satisfactory medical support. It also suggests an association of these issues with stigma and discrimination relating to mental illness which also gives the clients the feeling of what has been described as an absence of a 'win, win situation'. Another example of this view is included in Brian's statement in which he expressed that there was a low likelihood that clients who are recovering would be able to meet the expectations of achieving employment, as they also have to focus on getting well whilst exploring their employment prospects.

"Because you know just as any healthy you know mentally stable person can be all sorts of different people they seem to be putting all the mental health sort of down route to this kind of average and it's not going to be...I mean most people are just not going to be shop cashiers or whatever when they're, when they're well. They're going to be creative people or constructive people or social and there doesn't seem to be any option other than get well". (FG1).

This also indicates that clients focus on regaining their mental health as a priority while they also have interest in regaining some gainful employment. However, the latter is hampered by the illness experience. 
The LOC in this regard also seem to be external, as the clients seem to be grappling with both trying to remain mentally stable while going through the expectation that he/she also need to get into employment. This is further expressed in the majority of the participants' description of feeling vulnerable to expectations that they seek employment, as it is a condition for receiving social support that they demonstrate that they are actively seeking employment. This condition has also been expressed as exerting pressure on them in their recovery journeys. An example of this view is contained in Carla statement in which she described a sense of frustration at being asked to 'go to the job centre' while she was only becoming stable and having a bit of 'control':

"That's it. Just get well and once you're a little bit well, once you have a little bit of control it's like "ok, go to the job centre, there are the jobs there so go and mess up your life..." and then you're back to square one". (FG2).

This position also suggests that the clients is likely to feel a state of externality in LOC as a result of the expectation that they should be seen to be actively looking for employment after discharge, even when they did not feel that they were in enough control to handle the situation of seeking employment or even being able to secure or retain it. It also suggests that the working of the benefit system may have some negative impact on the post-hospital experiences, as the participants have also articulated that cuts in social welfare negatively affect their recovery.

This perception of externality is also further expressed from the point of view that the clients' sense of externality in LOC is indicated in a sense of dependency on the state and the conditions that have to be met to receive benefits to survive financially. An example of this view is highlighted by Trish, who described the procedure and conditions that accompany receiving State support, and describing the fear of ending up 'in trouble' as a result of failure to comply with the conditions:

"You have to claim benefits and in order to claim benefits ... and in order to claim benefits you bave to comply with job centre, you have to comply with your GP, you have to go to the GP and the psycbiatrist to get the letters to support your claim... you have to do everything othervise you just end up in trouble". (FG2).

This seems to suggest that there is the existence of underlying fear of failure to comply with conditions of receiving benefits, which could lead to being denied financial support and consequently not being in control over their finances. The externality in LOC is also suggested here due to the expressed risk of benefits sanctions, as opposed to the view of internality that accompany discharge from hospital.

The majority of the participants also expressed feeling dependent on the State due to unemployment, which they also see as intruding into their ability to control their lives. An example of this is included in Dwayne's statement in which he described that his mental illness experience has led to him having no other choice than to be dependent on state welfare. He also described this condition as constituting 'interference' in his life:

'Well I am dependant on the government for my finance, for my benefits and I'm dependant on the government for my medication which I need. So, society plays a big interference in my life...". (1-1).

Another example of this view of dependency due to reliance on state benefits is indicated in Ryan's statement in which he describes LOC from the parlance of 'limitation to the control' he has on his life:

"On the other side of things, there is a limitation to the control that I have because, you know, been on Benefits to take somebody else's money. Somebody else kind a make that decision as how much you can get. So, you don't have kind a have control because of my mental illness. You have kind a limited by the way that I can live my life. So, that put a limitation. So you can't live the way you, that you intend to your full potential. Also, I am living in rented accommodation, supported bousing. I am kind a living in somebody else's property. So you are living by somebody else's standards and not your own. You are not living in an environment that you would ideally choose to live. So, there is always that kind of limitation. At the same time, it kind a sits within me to break free of all that. Kind of live life more in a way that I want to where you know, living to my full potential, doing my own thing". (1-1).

This narrative describes factors of externality of LOC from mental health context, including other people making decision on one's behalf, and sometimes having to 'live in somebody else's standards', having no control as a result of mental illness experience, and a feeling that one is not living in an accommodation setting of their choice. The desire to 'break free' to 'live to own potential' and do one's own thing, as 
expressed here also indicates a realisation of the externality in LOC in the mental health context whilst recognising the challenges in this regard.

Patrick also described a feeling of partial level of control over his life. This was expressed in the context that the sense of incomplete control was due to not having his own home, as he is in supported housing and receiving benefits, which he described as being at the mercy of other entities:

"... but I also think that not feeling any control kind of keeps it perpetuating further because yes in a lot of ways I do feel ummm kind of control or are or kind of at the mercy of others. I mean because kind of you know I mean I live in...I'm on benefits, I'm on supported bousing and so you're kind of living on somebody else's money and somebody else's property. It's like you know... that in order to live..." (FG1).

These further suggests that there is some element of externality in LOC expressed by most of the participants, as they felt that they were unable to exert the type of control they would have wanted over their lives due to the influence of others. The externality also seems to be ongoing in their post hospital experiences of mental health.

\section{Discussion}

The findings suggest that the existence of several shifts in the mental health LOC of the participants were in correlation to the stages of their mental illness experiences. This includes the periods before the commencement of mental illness experience, the admission process that preceded hospitalisation, the inhospital experiences, discharge experiences and conditions that accompanied their discharge, and their experiences after discharge from hospital. These stages are characterised by different phases of the Internality and Externality (I-E).

The seemingly contradictory status of both internality and externality in LOC in the post-discharge experiences of most of the participants is a major finding in this study. This is highlighted in the shift between a feeling of a sense of control from perceived sense of freedom which arose from being discharged from hospital (internality of LOC) to a feeling of a lack of control due to perceived sense of inability to influence decisions or lack of control over their post-discharge care, (LOC externality). This suggests what has been termed the phenomenology of temporality (Aguiar de Sousa, 2017), as the internal-external LOC status of the study participants can be described as temporal in nature, and had been dictated and influenced by time, stages and differences in perceptions of control at those stages of mental health experiences. It has also been suggested that "meaning, sense and reason are intrinsically temporal" and that "point of view" is itself temporal." (Aguiar de Sousa, 2017). This may partially explain the combination of both internality and externality demonstrated in the current findings of the post hospital experiences of the majority of the study participants. It has also been articulated that the perception of an issue is always characterised by 'a double horizon', which includes 'the immediate past' and 'the immediate future', as well as 'a future horizon'. It also suggests that the spectrum of internal-external LOC status is not only subject to temporality as their LOC are subject to a passage between internal and external due to the impact of their experiences and the consequent perception of those experiences over time. The notion of temporality which states that the elements of impression leads on to retentions as well as what has been described as a 'retention of a retention' also seem to apply in the experiences of the study participants, as they seem to negotiate the levels of internality and externality in their experiences. The perception of a lack of control (externality) during hospital admission, treatment and subsequent discharge could lead to retention of such immediate past experiences after discharge, which may in turn lead to protension, an expectation of 'what is to come' (Aguiar de Sousa, 2017). The fear of repeated admissions expressed by the majority also seems to indicate an example of this phenomenon leading to a continued level of LOC externality which is occasioned by fear.

\section{Perception as a 'continuum' of present, past and future}

\section{Impression}

Retention
Protention 
In exploring Merleau-Ponty's views on temporality and subjectivity in the Phenomenology of perception, Aguiar de Sousa (2017) suggests that: "Whenever we perceive something, we are always originally directed to a future horizon. Thus, the present phase of perception is always surrounded by a double horizon, one side corresponding to the immediate past, the other to the immediate future" (Aguiar de Sousa (2017: 13). The paradox indicated in this phenomenon of temporality highlights the extent to which the participants navigate the trajectories of possible rehospitalisation due to a relapse, which indicates that there is a likelihood of a repeat of the cycle of internality to externality and back to internality in their MHLOC. It also further highlights the impact of expectations or fear of relapse and hospital readmission expressed by many of the participants in this study, which in turn impact on their perception of externality in LOC.

The seeming conscious effort also expressed by the participants as being made to prevent relapse and the consequent readmissions also seem to suggest that some level of LOC internality is being demonstrated by the participants in their bid to exert control over their lives, as well as to avoid perceived loss of control due to relapse. It also suggests that if the fear of what is to come becomes a reality over time in terms of relapse and subsequent readmission, the clients' expectations (protention) is likely to be further accentuated, as they are likely to expect a repeat of their experiences under the impression as well as the retention stages. As literature suggests that the level of internality in the LOC of mental health clients is a determining factor in their engagement with services (Treacy et al, 2015), and the tendency of individuals in the internality spectrum to seek medical care in comparison to those in the externality spectrum (Kesavayuth et al, 2020); it proposes also that focusing on such factors identified as likely to increase internality are also likely to impact on the perception of service users on the internal-external (I-E) spectrum.

The foregoing suggests that suitable interventions by mental health services towards avoiding repetition of negative experiences for clients are likely to reduce the fear and anxiety of what is to come, which is an element of LOC externality (a fear of the future). Examples of such interventions include those that work with clients on self-identified goals to build their independence, functionality, and wellbeing in the community (Treacy et al, 2015). Furthermore, the highlighted relationship between collectivism and externality in LOC for Black and Minority Ethnic communities (Papadopoulos, 2009; Cheng et al, 2013; Joseph, 2018), suggests the high desirability in introducing service-user led, peer support, and culturally sensitive programmes that focuses on the needs of the BME communities with experience of mental health challenges. They also include programmes aimed at reduction in inequality in access to employment, in view of the identified intersection of financial challenges, internalised locus of control and mental health (Frankham et al, 2020). As it has also been established that the quality of healthcare is 'significantly predicted' by "patients' ethnicity, language and culture" (Chauhan et al, 2020:119), community programmes that recognises the importance of and are tailored to promoting culture and identity for the targeted groups are highly desirable (Joseph, 2018). These programmes can also be tailored to encourage clients to be active participants and take ownership to build their sense of belonging, self-esteem, and promote hope for the future, which are prerequisites for LOC internality (Joseph, 2018). Such interventions are therefore likely to increase the level of internality in their mental health LOC, reduce anxiety and minimise relapse episodes or incidents.

\section{Conclusion}

This study has explored the participants' responses to the interview questions in relation to their perception of control of their lives. Although not generalisable to the A \& C populations with experience of mental health challenges and services, this exploration has thrown more light to the perceived reasons for the externality in their LOC- which has now been identified as due to a combination of factors including their own experiences of social stressors which led to mental health challenges and breakdown. Other factors include issues related to assessment, hospital admission, treatment, discharge and after discharge stages of their experiences with mental health services, which were perceived to have been driven by experiences of racial discrimination and other social inequalities. The study also enabled the 'world view' of the study participants to be explored, using the idiographic approach of the Interpretative Phenomenological Analysis 
(IPA). The researcher has also been able to rely on the participants' expression of their own perception from their own experiences to describe as well as interpret those expressions. The expressions of seemingly contradictory LOC within the context of the out of hospital experiences of the participants also raise the phenomenon of temporality in the LOC of the participants, as it highlights that different experiences impacted on their LOC, including the temporality in their internality and a sense of a continuation of externality in LOC in their post hospital experiences. It also raises the need to address the differences in their perception within the same contexts and suggests the necessity to understand what factors may contribute to service users' externality with a view to reducing or addressing them in order to place them on a higher internality scale. These original findings which also points to temporality also present a novel start to a band of knowledge that is required into exploring the explanatory model for the experiences of this study populations in mental health settings in the United Kingdom, as there had been no known study which has explored these issues with mental health clients in general and African and African Caribbean groups in particular, in spite of the over representation of the study populations group in mental health settings in the United Kingdom. The findings may not only be useful for supporting peoples of other ethnic communities, but applicable to all mental health service consumers in general.

\section{Declarations}

\subsection{Informed consent}

The authors declared that no conflict of interest exist in this study.

\subsection{Competing Interests}

The authors declared that signed informed consent was obtained from all participants in this study.

\section{How to Cite this Article:}

Joseph, S., \& Keating, F. (2021). Self-control and Mental Health? Exploring Perceptions of Control from the Experiences of Black and Minority Communities. Advanced Journal of Social Science, 8(1), 145-163. https://doi.org/10.21467/ajss.8.1.145-163

\section{References}

Aguiar de Sousa, L. (2017) Merleau-Ponty on Temporality and Subjectivity in the Phenomenology of Perception. Available at: https://www.academia.edu/Documents/in/Phenomenology_of_Temporality

Arain, M., Campbell, M.J., Cooper, C.L., \& Lancaster, G. A. (2010) 'What is a pilot or feasibility study? A review of current practice and editorial policy’ BMC Medical Research Methodology 10 (67): 1-7

Borrell-Carrió, F., Suchman, A. L., and Epstein, R. M. (2004) 'The Biopsychosocial Model 25 Years Later: Principles, Practice, and Scientific Inquiry'. The Annals of Family Medicine 2 (6), 576-582

Brocki, J. M. and Wearden, A. J. (2006) 'A Critical Evaluation of the use of Interpretative Phenomenological Analysis (IPA) in Health Psychology'. Psychology \& Health 21 (1), 87-108

Care Quality Commission, 2014; Care Quality Commission (2014) Monitoring the Mental Health Act in 2012/13.: Care Quality Commission. Available from: http://www.cqc.org.uk/sites/default/files/documents/cqc_mentalhealth_2012_13_07_update.pdf.

Chauhan, A., Walton, M., Manias, E. et al (2020). The safety of health care for ethnic minority patients: a systematic review. Int J Equity Health 19,118

Cheng, C., Cheung, S., Chio, J. H., and Chan, M. S. (2013) 'Cultural Meaning of Perceived Control: A Meta-Analysis of Locus of Control and Psychological Symptoms Across 18 Cultural Regions'. Psychological Bulletin 139 (1), 152-188

Clarke, C. (2009) 'An introduction to interpretative phenomenological analysis: a useful approach for occupational therapy research' British Journal of Occupational Therapy, 72, (1): 37-39

Crisp, N., Smith, G. and Nicholson, K. (2016) Old Problems, New Solutions: Improving Acute Psychiatric Care for Adults in England: The Commission on Acute Adult Psychiatric Care] available from http://media.wix.com/ugd/0e662e_aaca63ae4737410e9e2873dfde849841.pdf

Darawsheh, W (2014) 'Reflexivity in research: Promoting rigour, reliability and validity in qualitative research' International Journal of Therapy and Rehabilitation 21 (12), 560-568

Department of Health (2013) Annual Report of the Chief Medical Officer 2013, Public Mental Health Priorities: Investing in the Evidence. Department of Health Available from

https://www.gov.uk/government/uploads/system/uploads/attachment_data/file/413196/CMO_web_doc.pdf

Edge, D. (2011) 'It's leaflet, leaflet, leaflet then, "see you later"': black Caribbean women's. Perceptions of perinatal mental health care' British Journal of General Practice, 61: 256-262.

Elder, R., Evans, K., Nizette, D. (2009) Psychiatric and Mental Health Nursing. 2nd edn. Australia.: Elsevier 
Engel, G. L. (2004) The need for a new medical model: A challenge for Biomedicine, in Caplan, A.L., McCartney, J.J., \& Sisti, D.A (2004) Health, Disease and Illness, Concepts in Medicine Georgetown University Press, U.S.A. Chapter 7, Pages 51-64.

Fearon, P., Kirkbride, J. B., Morgan, C., Dazzan, P., Morgan, K., Lloyd, T., Hutchinson, G., Tarrant, J., Fung, W. L. A., Holloway, J., Mallett, R., Harrison, G., Leff, J., Jones, P. B., and Murray, R. M. (2006) 'Incidence of Schizophrenia and Other Psychoses in Ethnic Minority Groups: Results from the MRC AESOP Study'. Psychological Medicine 36 (11), 1541-1550

Fernando, S. (1998). Studies into issues of 'race' and culture in psychiatry. Psychological Medicine 28: 496-497

Fernando, S., \& Keating, F. (2009) Mental Health in a Multi-Ethnic Society: A Multidisciplinary Handbook. Hove: Routledge

Frankham, C., Richardson, T. \& Maguire, N (2020). Do Locus of Control, Self-esteem, Hope and Shame Mediate the Relationship Between Financial Hardship and Mental Health?. Community Ment Health, 56, 404-415.

Garcia-Toro, M. \& Aguirre, I. (2007) 'Biopsychosocial model in Depression revisited. Medical Hypotheses 68: 683-691

Hill, D.J. \& Bale, R.M. (1980) 'Development of the Mental Health Locus of Control and Mental Health Locus of Origin Scales' Journal of Personality Assessment 44 (2), 148-156

Iqbal, Neelam. (2021 Minority Mental Health: Intersections between Faith and Ethnicity). http://dx.doi.org/10.2139/ssrn.3767398

Joseph, S. (2018) Exploring the relationship between Locus of Control and the perception of the quality of mental health care experienced by African \& Caribbean service users. Thesis or dissertation. Available at: pure.royalholloway.ac.uk

Keating, F, Robertson, D., McCulloch, A., Francis, E. (2002) Breaking the Circles of Fear: A Review of the Relationship between Mental Health Services and African and Caribbean Communities. London: The Sainsbury Centre for Mental Health

Keating, F. (2016) 'Racialised Communities, Producing Madness and Dangerousness'. Intersectionalities: A Global Journal of Social Work Analysis, Research, Polity, and Practice 5 (3), 1-13

Kesavayuth, D.; Poyago-Theotoky, J.; Zikos, V. (2020), Locus of control, health and healthcare utilization. Econ. Model. 86, 227-238

Kitzinger, J. (1995) 'Qualitative Research - Introducing Focus Groups’ British Medical Journal 311: 299-302.

Knight, M.T.D., Wykes, T., and Hayward, P. (2003) 'Groups don't understand': an investigation of stigma in schizophrenia using interpretative phenomenological analysis (IPA)’. Journal of Mental Health 12 (3), 209-22

Lambert, S. D, and Loiselle, C. G, (2008) 'Combining individual interviews and focus groups to enhance data richness' Journal of Advanced Nursing 62 (2), 228-237

Mantovani, N., Pizzolati, M., and Edge, D. (2017) 'Exploring the Relationship between Stigma and Help-Seeking for Mental Illness in African-Descended Faith Communities in the UK'. Health Expectations: An International Journal of Public Participation in Health Care and Health Policy 20 (3), 373-384

Morling, B., \& Kitayama, S. (2008) Culture and motivation. In J.Y. Shah \& W. L. Gardner (Eds.), Handbook of motivation science (pp. 417-433). New York, NY: Guilford Press.

Mulveen, R. and Hepworth, J. (2006) 'An Interpretative Phenomenological Analysis of Participation in a Pro-Anorexia Internet Site and its Relationship with Disordered Eating'. Journal of Health Psychology 11 (2), 283-296

National Health Services, NHS (2013) The Six Caldicott Principles. Available at:

www.connectingforhealth.nhs.uk/systemsandservices/infogov/caldicott

Papadopoulos, C. (2009). Stigma towards groups with mental health problems: an individualism-collectivism cross-cultural comparison. A thesis submitted in partial fulfilment of the requirements of Middlesex University for the degree of Doctor of Philosophy. Accessed 4th February 2014 from: British Library Electronic Theses Online Service- EThOS.

Prince, M., Stewart, R., Ford, T. \& Hoptopf, M. (2003) Practical psychiatric epidemiology. Oxford.

Proto, E \& Quintana-Domeque, C. (2020). COVID-19 and Mental Health Deterioration among BAME Groups in the UK. Available at: https://www.cesifo.org/en/publikationen/2020/working-paper/covid-19-and-mental-health-deterioration-among-bame-groups-uk

Redsell, S.A., \& Cheater, F.M. (2001) 'The Data Protection Act (1998): implications for health researchers. In Methodological Issues in Nursing Research' Journal of Advanced Nursing 35 (4): 508-513.

Ruiz-Casares, M. (2014) 'Tailor informed consent processes' Nature (513): 304

Smith, J. \& Osborne, M. (2003). Interpretive phenomenological analysis. In J.A. Smith (Ed.) Qualitative psychology: A practical guide to research methods (pp.51-80). London: Sage.

Smith, J.A., Flowers, P., \& Larkin, M. (2012) Interpretative Phenomenological Analysis: Theory, Method and Research. London: SAGE Publications

Smith K, Bhui K, Cipriani A. COVID-19, mental health and ethnic minorities. Evidence-Based Mental Health. 2020 Aug 1; 23(3):89-90.

Smyth, G., Harries, P., \& Dorer, G. (2011) 'Exploring mental health service users' experiences of social inclusion in their community occupations' British Journal of Occupational Therapy 74 (7): 323-331

Starks and Trinidad, 2007 Starks, H. and Trinidad, S.B. (2007) 'Choose your method: A comparison of phenomenology, discourse analysis, and grounded theory'. Qualitative health research, 17 (10): 1372-1380

Stewart and Shamdasani, 1990 Stewart, D.W. \& Shamdasami, P. N. (1990) Focus Groups: Theory and Practice. London: Sage

Teijlingen, E V., \& Hundley, V. (2002). The importance of pilot studies. Nursing Standard. 16, (40):33-36.

Thabane, L., Ma, J., Chu, R., Cheng, J., Ismaila, A., Rios, L. P., Robson, R., Thabane, M., \& Goldsmith, C. H. (2010): A tutorial on pilot studies: The what, why and How. BMC Medical Research Methodology 10 1. Available at: https://doi.org/10.1186/1471-2288-10-1

Treacy, S., Reidy, H., \& Webber, M. (2015) Evaluation of the Croydon Reablement Service. International Centre for Mental Health Social Research, Department of Social Policy and Social Work, University of York. Available at: https://www.york.ac.uk/media/spsw/documents/cmhsr/CroydonReablementEvaluation2015.pdf 
Stephen Joseph and Frank Keating, Adv. J Social Sci.; Vol. 8, Issue 1, pp: 145-163, 2021

Williams, A., \& Katz, L. (2001) 'The Use of Focus Group Methodology in Education: Some Theoretical and Practical Considerations' International Electronic Journal for Leadership in Learning 5: (3)

Willig, C. (2003) Introducing qualitative research in psychology: adventures in theory and method. Open University Press.

Publish your research article in AIJR journals-

Online Submission and Tracking

$\checkmark$ Peer-Reviewed

$\checkmark \quad$ Rapid decision

$\checkmark \quad$ Immediate Publication after acceptance

$\checkmark \quad$ Articles freely available online

$\checkmark \quad$ Retain full copyright of your article. Submit your article at journals.aijr.org
Publish your books with AIJR publisher-

$\checkmark \quad$ Publish with ISBN and DOI.

$\checkmark \quad$ Publish Thesis/Dissertation as Monograph.

$\checkmark \quad$ Publish Book Monograph.

$\checkmark \quad$ Publish Edited Volume/ Book.

$\checkmark \quad$ Publish Conference Proceedings

$\checkmark$ Retain full copyright of your books.

Submit your manuscript at books.aijr.org 